Bio - grafía. Escritos sobre la Biología y su Enseñanza. ISSN 2027-1034

Edición Extraordinaria. p.p. 1125 - 1136

Memorias del IX Encuentro Nacional de Experiencias en Enseñanza de la Biología y la Educación Ambiental. IV Congreso Nacional de Investigación en Enseñanza de la Biología.

\title{
LA EPE: UNA ESCUELA EN EL CORAZON DE LA MONTAÑA
}

Rosa Galindo

Paula Salcedo

Raquel Soto

"El texto cuya lectura es como un perderse en un bosque, en caminos que no conducen a ninguna parte. Irrupción de lo inesperado. Milagro del nacimiento" (Melich 1996,p.275 )

\section{RESUMEN:}

Esta ponencia tiene como apuesta mostrar cómo el aprendizaje de la biología, el estudio de la vida y lo vivo va más allá del abordaje de aspectos disciplinares que están desligados de la vida cotidiana y que dejan de lado situaciones ambientales y sociales que permiten re-configurar nuestra relación con Gaia, esto implica no solo re-conocer nuestros problemas sino estar dispuestos a imaginar soluciones contextuales que tengan como base metodológica las Actividades de Totalidad Abierta (ATA's). En particular, queremos mostrar a través de este escrito la situación que hemos estado viviendo desde hace 8 años a causa de una montaña de escombros sobre la quebrada Puente Piedra (ubicada en la subcuenca del salitre) nos mantiene en riesgo permanente, esto nos ha llevado a abrir diferentes espacios para abordar esta problemática desde el diálogo y la reflexión, aspectos que han dado lugar a que los estudiantes y toda la comunidad educativa se movilicen en pro de búsquedas y aliados que posibiliten construir rutas de trabajo que permitan desarrollar acciones concretas. Éstas, son las palabras resultado de un largo camino de búsquedas, de altibajos, de logros, discusiones y acuerdos que han precisado momentos esperanzadores y de realidades en torno a esta "montaña" que hoy por hoy, crece y crece de forma exponencial.

PALABRAS CLAVE: montaña de escombros, aprendizaje de la biología, comunidad, quebrada, Actividades Totalidad Abiertas.

\footnotetext{
${ }^{1}$ Rosa Galindo, directora de la Escuela Pedagógica Experimental. Correo: rosa.galindo@epe.edu.co . Paula Salcedo, Docente de ciencias y de economía azul de la Escuela Pedagógica Experimental. Correo: paula.salcedo@epe.edu.co

Raquel Soto, Docente de ciencias de la Escuela Pedagógica Experimental. Correo: raquel.soto@epe.edu.co
} 
Bio - grafía. Escritos sobre la Biología y su Enseñanza. ISSN 2027-1034

Edición Extraordinaria. p.p. 1125 - 1136

Memorias del IX Encuentro Nacional de Experiencias en Enseñanza de la Biología y la

Educación Ambiental. IV Congreso Nacional de Investigación en Enseñanza de la

Biología.

\section{ABSTRACT:}

This paper is going to show like the learning of biology, the study of life go beyond than disciplinary studies, these were not connected with daily life and left aside environment and social aspects which are allow us re-configured our relationship with Gaia (earth), This implies not only recognizing our problems but also being willing to imagine contextual solutions that have as a methodological basis the Actividades de Totalidad Abierta (ATA's). In particular, in this paper we want to show a situation that we have been living by 8 years, a big debris's mountain near the Puente Piedra's ravine (located in Salitre's subbasin) is a permanent risk to us, this situations was allowed us work in different spaces of dialogue and reflection where the students and community united have looked different work forms to develop concrete solutions to change that conditions. These words are the result of a long search process, of ups and downs, of achievements, discussions and agreements that have given hopeful moments and realities around this "mountain" that nowadays, grows and grows exponentially.

KEY WORDS: Mountain of debris, biology learning, community, ravine, actividades totalidad abierta.

\section{INTRODUCCIÓN:}

La Escuela Pedagógica Experimental celebra la alegría de existir para conmemorar 40 años de vida que se configuran como una apuesta que nos conecta con la Madre Tierra como un tejido que busca reconocer la incesante labor de las manos de quienes han sido protagonistas por cuatro décadas, dado que conlleva creación, re-unión, movimiento, deconstrucción, intercambios y trueques de palabras, memorias y sentires.

Esta conexión se gesta porque la EPE está incrustada en la montaña, rodeada de árboles donde anidan diversos pájaros que con su canto mañanero saludan cotidianamente a la comunidad educativa. En medio de este bosque nublado y húmedo yacen las hojas y las ramas de las moras silvestres donde habitan unos seres alados, de color verde y naranja que han sido llamados diablitos, que se han convertido en el símbolo de pertenencia a la EPE.

Si se decide avanzar más, encontramos un arboreto donde habita una colección de distintas especies nativas de la zona como: laurel huesito, mano de oso, tuno, chilca, mortiño, acacia, encenillo, sangre de drago, líquenes y hongos que se nutre de la quebrada Puente de piedra en la que se dio un proceso de recuperación gracias al empeño de Conservación internacional $(\mathrm{Cl})^{2}$, el barrio y la escuela.

Esta situación fue tomada como punto de partida en el año 2012 para iniciar un proyecto de recuperación de la quebrada, que nos ha permitido partir de una necesidad presente

\footnotetext{
2 Conservación Internacional (Cl): ONG encargada de velar por una convivencia sostenible entre el los humanos y el ambiente, enf ocada a trabajar con gobiernos para asegurar que tengan el conocimiento y herramientas para aplicar políticas que sean buenas respons ables y protejan nuestra riqueza natural. Recuperado de: http://www.conservation.org.co/ 24 de may o de 2017.
} 


\section{Bio - grafía. Escritos sobre la Biología y su Enseñanza. ISSN 2027-1034}

\section{Edición Extraordinaria. p.p. 1125 - 1136}

Memorias del IX Encuentro Nacional de Experiencias en Enseñanza de la Biología y la

Educación Ambiental. IV Congreso Nacional de Investigación en Enseñanza de la Biología.

en el contexto a conocer más nuestro entorno desde los contenidos biológicos (organismos presentes, redes tróficas, calidad del agua, procesos de recuperación de los ecosistemas...etc) y así generar un sentido de apropiación por parte de los estudiantes hacia su territorio, como lo propone Velasco (2001).

Dentro de este proyecto, los lazos generados con la comunidad propiciaron que los estudiantes visibilizarán la quebrada (jornadas de limpieza y embellecimiento), hicieran procesos de reforestación de la zona de ronda a partir de diseños florísticos, identificaran su ubicación en el corredor de paramos y su importancia dentro de la macro-estructura ecológica e hídrica de Bogotá, analizaran por medio de bioindicadores los niveles de contaminación... entre otras acciones reportadas por García (2013).

Sin embargo, este escenario se ha transformado ya que a partir de los años 2009 y 2012 la quebrada Puente Piedra, ubicada al lado de nuestra institución, empezó a contaminarse de forma abrupta a causa del vertimiento de escombros por parte de la comunidad. Como consecuencia (...) su cauce fue desplazado 7 metros hacia la escuela (...) a partir de ello, el estudiante Leonardo Segura quien cursaba el nivel $11\left(\right.$ Grado $\left.9^{\circ}\right)$ realizó el monitoreo de los niveles de contaminación del agua presentes allí (Ruiz, Granados \& Soto, 2016 P. 2)

Estos escombros fueron formando una montaña actualmente considerada como una amenaza para la comunidad EPE en la medida que su crecimiento se ha dado de manera exponencial en los últimos años, y junto con las fuertes lluvias, han generado alteraciones en las condiciones hídricas y de suelos de estos ecosistemas dando lugar a considerar este espacio de la escuela como una zona que está en peligro de ser cobijada por la montaña de escombros.

Desde la perspectiva política y normativa a nivel nacional e internacional, existen varias legislaciones que velan por el respeto y el cuidado de la naturaleza y rechazan este tipo de acciones o actividades que puedan generar altos impactos negativos sobre este lugar, claro ejemplo de ellos son:

1. constitución política de Colombia (Capitulo 3, art. 79) promulga:

"Todas las personas tienen derecho a gozar de un ambiente sano. La ley garantizará la participación de la comunidad en las decisiones que puedan afectarlo. Es deber del Estado proteger la diversidad e integridad del ambiente, conservar las áreas de especial importancia ecológica y fomentar la educación para el logro de estos fines." (P. 25)

2. El fallo del consejo de estado sobre los cerros orientales y la sentencia del río Bogotá en el Acuerdo 30 de 1976 (30 de septiembre), declaró los cerros como "Área de Reserva Forestal Protectora" "la zona denominada Bosque Oriental de Bogotá". 


\section{Bio - grafía. Escritos sobre la Biología y su Enseñanza. ISSN 2027-1034}

\section{Edición Extraordinaria. p.p. 1125 - 1136}

Memorias del IX Encuentro Nacional de Experiencias en Enseñanza de la Biología y la

Educación Ambiental. IV Congreso Nacional de Investigación en Enseñanza de la Biología.

3. Acuerdo por el que se prorroga y modifica el Acuerdo sobre Planificación de Aprovechamiento y utilización de Áreas Forestales Tropicales, del 17 de agosto de 1978.

Pese a existir dicha legislativa, es notorio respecto a la problemática que afronta la escuela, que existen muchos actores que hallan la manera de pasar por encima de estas leyes sin reflexionar la importancia de estas áreas de reserva por su valor intrínseco además de los bienes y servicios que nos proporcionan. Por esto mismo es que el aprendizaje de la biología se convierte en un punto de partida para entrar a reflexionar los modos en que se puede entrar a comprender, problematizar y plantear posibles soluciones ante las dinámicas ambientales y ecológicas que emergen de la situación de esta montaña. Para ello, se propone abordar el problema de forma holista, donde diferentes campos de saber y de poder entran a movilizar el cuidado y detrimento de este problema causado por acciones antrópicas.

Por tanto, la presente ponencia tiene por objeto discutir como el tratamiento de las problemáticas socio-ambientales desde el aula, lo lleva a trascender más allá de las cuestiones meramente disciplinares para aportar en la transformación del contexto desde acciones reales que involucren no solo a la escuela sino también a distintos actores de la comunidad y entidades estatales comprometidas con el territorio.

\section{METODOLOGÍA:}

Nuestra apuesta permanente como maestros en la EPE ha sido construir propuestas que articulen la escuela con el contexto con la intención de establecer un entramado de relaciones que busca vincular el aprendizaje escolar con la educación para la vida, sin perder de vista que deben estar relacionadas con el contexto cultural de los niños y los jóvenes para que resulten valiosas y pertinentes. Para ello se ha retomado como metodología de trabajo las Actividades Totalidad Abiertas ATA's que busca lograr una tensión cognoscitiva- afectiva entre el individuo-colectivo y la actividad. (Pedreros R., Velasco A., Leuro R. Arcos F., Hernández G. Molina A. \& Segura D, 1995; Molina A, Pedreros R. \& Segura D. 1997).

La propuesta metodológica e la escuela está centrada en generar un ambiente de trabajo donde emerjan diversas formas de explicar, predecir e interpretar de los estudiantes ante una pregunta, situación problemática o fenómeno de manera que se avance en una dinámica en la que el estudiante siempre sabe lo que está haciendo y por qué lo está haciendo, esta búsqueda permite y propicia que en las interacciones de grupo aparezcan nuevas preguntas e inquietudes que generan otras búsquedas en una dinámica recurrente (Segura, 2007).

Esta recurrencia es la que configura en la EPE los proyectos como una posibilidad para pensar lo local en la escuela que conlleva a reflexionar sobre diferentes aspectos que atraviesan la vida, la cotidianidad de sus habitantes y su relación con el medio promoviendo prácticas de sostenibilidad (entendidas como las acciones que buscan satisfacer las necesidades de las generaciones presentes respetando los ecosistemas 


\section{Bio - grafía. Escritos sobre la Biología y su Enseñanza. ISSN 2027-1034}

\section{Edición Extraordinaria. p.p. 1125 - 1136}

Memorias del IX Encuentro Nacional de Experiencias en Enseñanza de la Biología y la

Educación Ambiental. IV Congreso Nacional de Investigación en Enseñanza de la Biología.

naturales y la calidad del medio ambiente, para no comprometer de manera negativa las futuras generaciones). Es desde aquí, que nos hemos ido acercando a conocer, discutir y develar los problemas de nuestro país en asuntos relacionados con la soberanía alimentaria, el territorio, los agrocombustibles, el manejo de las fuentes hídricas, la explotación minería, el proceso de paz, que nos atraviesan como seres humanos, no solo en el marco de los discursos sino en el nivel de las decisiones y posibilidades de construcción de sí mismos como comunidad, territorio y región.

De esta manera, se intenta reconocer los roles que podría jugar nuestra participación como escuela en la construcción de iniciativas colectivas e individuales con la intención de aproximarnos a situaciones concretas para conocer nuestro país desde la construcción de problemas que son sentidos por nuestras comunidades porque nos afecta de manera permanente, esto ha implicado generar un compromiso de la escuela con los problemas contemporáneos que nos llevaron en un primer momento a iniciar procesos de recuperación de la quebrada Puente de Piedra a través de alianzas con la comunidad y con diversas organizaciones y en un segundo momento a trabajar nuevamente en conjunto en pro de buscar soluciones frente a la montaña de escombros que ha crecido de manera exponencial y que amenaza nuestra comunidad estudiantil.

En esta búsqueda los jóvenes de los grados 9, 10 y 11 que han estado participando en este proyecto, enmarcado en la aplicación pedagógica de la propuesta de Economía Azul (Segura, Díaz \& García, 2015), se convirtieron en protagonistas de su hacer, ganaron reconocimiento, pues aprendieron a confiar en sus propias posibilidades, construyeron hábitos de trabajo y avanzaron en la construcción de su conocimiento. También se logró favorecer la comprensión de diversas situaciones de clase ya que éstas tienen sentido para él, por ello las actividades que se proponen les permite reconocerse, confrontarse y auto criticarse de manera constructiva.

Dentro de las actividades que se han de abordar, se toma como principal referente la montaña de escombros, una "montaña problema" que está inmersa en el contexto de los estudiantes y de toda la comunidad educativa, pues pone en riesgo las dinámicas que se dan a diario en la escuela por su inestabilidad topográfica, geológica, química y biológica. Para ello, se propone un ejercicio de reflexión, crítica y análisis en torno a esta montaña, aspecto que da lugar a realizar una aproximación de saberes en torno al conocimiento disciplinar y al conocimiento común, considerados desde esta perspectiva como una relación que constantemente va hilando un tejido y por tanto, no se puede desligar de aquellas realidades de comprensión y problematización.

Es así, que se realizan encuentros de debate, discusión, foros informativos, prácticas de laboratorio y experimentales, camitas ecológicas, estudios de micro relieve, topográficos y de bioindicadores del suelo y la montaña, con el firme propósito de construir una ruta de argumentos y de saberes que permitan comprender la incidencia biológica y ecosistémica que tiene esta montaña de escombros en la escuela y en los Cerros Orientales de Bogotá. 
Bio - grafía. Escritos sobre la Biología y su Enseñanza. ISSN 2027-1034

Edición Extraordinaria. p.p. 1125 - 1136

Memorias del IX Encuentro Nacional de Experiencias en Enseñanza de la Biología y la

Educación Ambiental. IV Congreso Nacional de Investigación en Enseñanza de la

Biología.

\section{RESULTADOS:}

Inicios del proyecto: El proyecto inició sus acciones en pro de la recuperación de la quebrada puente piedra basadas 6 ejes de trabajo (García-Mayorca, Lujan, Arbeláez, Fernández, Velazco \& García, 2014) apoyados por la comunidad y por entidades como Conservación internacional, ACUALCOS ${ }^{3}$, Jardín Botánico, Secretaria de ambiente, donde fue posible la realización de diversas acciones en pro de mejorar el estado de contaminación de la quebrada (García, 2013) y por medio de los cuales se abarcaron distintos conceptos disciplinares (biología, geografía y sociales) que se articularon de la siguiente manera (Ver anexo 1).

La Situación actual de la Quebrada Puente Piedra: A pesar de los innumerables esfuerzos y trabajo desarrollados por los estudiantes del proyecto para recuperar la quebrada y disminuir los niveles de contaminación, constantemente los volqueteros siguieron haciendo vertimientos hasta formar una gran montaña de escombros "entre los que se encuentran escombros, material de excavación, basuras, entre otros, específicamente encontramos, retales de ladrillo, cerámicas, tubería de PVC, vidrio, concreto, metal, recebo, podas de vegetación, tierra o suelo orgánico, etc." (CAR, p. 4); ésta ha sido denunciada ante diversas organizaciones que abogan por el cuidado del ambiente, a diferentes medios de comunicación y a instancias policiales sin encontrar una solución expedita ante esta problemática que hace varios años nos está afectando como lo muestra la carpeta del proceso que se ha gestado desde la EPE donde se encuentra un registro pormenorizado de los documentos legales que demuestran las denuncias del depósito de escombros sobre la quebrada Puente Piedra ubicada sobre la sub-cuenca del salitre.

Sin embargo, desde los meses de mayo y junio del año 2016 la organización Conservación Internacional convocó a reunión a organizaciones como: ACUALCOS, Casa Taller Las Moyas ${ }^{4}$, La Mesa ambiental Hijos de las Moyas ${ }^{5}$, CIMAVISION $^{6}$, la EPE, la I.E.D. Campestre Monteverde, con el fin de generar una articulación entre las mismas para hacer frente a las problemáticas actuales que han obstaculizado los procesos de recuperación de las quebradas Morací y Puente Piedra y al mismo tiempo fortalecer los procesos de recuperación mencionados. A estas reuniones asistieron como representantes de la EPE un equipo interdisciplinar de maestros de la escuela y personal de mantenimiento.

\footnotetext{
${ }^{3}$ Asociación de servicios públicos comunitarios San isidro I y II, San Luis y la Sureña, El objetivo de la asociación es administrar el servicio público de agua y alcantarillado propendiendo en todo sentido por la salud de los Asociados y la correcta utilización de los servicios suministrados, promover la defensa y protección de los recursos hídricos, a trav és de la participación activ a y la educación de los Asociados. Promover campañas de reforestación para conservar las resenas y el entorno ambiental. Recuperado de: https://www.f acebook.com/pg/ACUALCOS-ESP-157630264306531/about/?ref=page internal 23 de mayo de 2017.

${ }^{4}$ Es un espacio de aprendizaje comunitario que recibe a niñas y niños del Barrio de San Luis diariamente fuera del horario escolar. En el espacio todos los participantes se desenenv uelv en con autonomía según sus necesidades. Cada día responde a las dif erentes propuestas de las niñas y niños, pudiendo realizarse desde actividades en la huerta, juegos colectivos, música o comidas en conjunto. Recuperado de: http://mapa.reev 0.org/reports/view/502 24 de may o de 2017

${ }^{5}$ Mesa ambiental hijos de las moy as, actores de la comunidad que se han organizado en pro de la protección del territorio y el cuidado del ambiente natural.

${ }^{6}$ es un canal de televisión Comunitario, que pretende ser un medio alternativo que genere y exprese la libertad de abrir espacios de comunicación, que promuev a la Equidad social, los Derechos humanos, la sustentabilidad ambiental y la riqueza cultural y las expresiones artísticas y sociales de comunidad y los cerros orientales de la UPZ 89 de la localidad de Chapinero, Bogotá-Colombia. Recuperado de: https://www.f acebook.com/pg/Cimav isionTelevision/about/?ref =page internal
} 


\section{Bio - grafía. Escritos sobre la Biología y su Enseñanza. ISSN 2027-1034}

\section{Edición Extraordinaria. p.p. 1125 - 1136}

Memorias del IX Encuentro Nacional de Experiencias en Enseñanza de la Biología y la

Educación Ambiental. IV Congreso Nacional de Investigación en Enseñanza de la Biología.

En las primeras reuniones se hicieron recuentos acerca de las problemáticas actuales (el depósito de escombros, de aguas residuales y el robo de cauce de la quebrada Morací), los esfuerzos e intentos fallidos llevados a cabo por las distintas instituciones con la intención de cortarlas de raíz por medio de distintos procesos legales. A partir de estos recuentos, se reúne todos los documentos y soportes que tienen ACUALCOS y La EPE de estos procesos, Conservación Internacional estudia los documentos y trae en las siguientes reuniones a un grupo de abogados que revisan toda la documentación y encuentran que en las mismas hay Resoluciones y querellas emitidas por la CAR a favor de la EPE para frenar y quitar definitivamente el depósito de escombros sobre la quebrada, pero estas no han sido ejecutadas. Frente a esta situación, se planteó iniciar procesos desde 4 perspectivas:

1. La acción de cumplimiento: entre los documentos legales de la escuela y de ACUALCOS se encontraron sentencias relacionadas con los problemas de aguas residuales y las zonas de reserva y amortiguación.

2. Derecho de petición: este proceso se adelanta por dos razones: la violación o vulneración de la seguridad de los niños por el concepto de los riesgos que generan los escombros en caso de avalancha, este derecho de petición fue firmado por toda la comunidad de la EPE y la segunda razón, una reclamación por detrimento patrimonial (teniendo en cuenta que en los procesos de recuperación hubo inversión de dineros).

3. Documento de rigor técnico: es un documento que se envió a la CAR como petición para que intervenga en las problemáticas presentes en las quebradas Puente Piedra y Morací; éste documento contiene además todo el concepto de los riesgos de los escombros en caso de avalancha.

4. Trabajo social: se socializó con la comunidad del barrio y de la escuela las problemáticas presentes y los procesos realizados para concientizarlos sobre las consecuencias que se pueden venir a partir de las acciones legales que se han realizado.

También se encontró el fallo del consejo de estado sobre los cerros orientales y la sentencia del río Bogotá ${ }^{7}$, que surgieron a partir de demandas realizadas por parte de la comunidad a causa de construcciones ilegales sobre los cerros orientales (I foro de Cerros orientales, 2016); por tanto se decidió tomar estos casos como referencia para aplicarlo en nuestro caso con la recuperación de las quebradas.

Para dar ejecución a los procesos anteriores, CIMAVISION asistió a la escuela, para realizar filmaciones de la quebrada y unas entrevistas a los estudiantes del proyecto acerca de la problemática del depósito de escombros (recuperado de: https://www.youtube.com/watch?v=bxeeidPuAcM); Se entregó a los abogados un documento que mostraba el número de estudiantes que hay en la EPE; Conservación internacional hizo el documento de rigor técnico, se realizaron derechos de petición por cada obligación que tiene cada ente, éstos fueron firmados por los representantes de

\footnotetext{
7 Acuerdo 30 de 1976 (30 de septiembre), por medio del cual el INDERENA declaró "Área de Reserva Forestal Protectora" "la zona denominada Bosque Oriental de Bogotá", y fue aprobada por el presidente de la república por medio de la Resolución 76 de 1977 (31 de marzo).
} 


\section{Bio - grafía. Escritos sobre la Biología y su Enseñanza. ISSN 2027-1034}

\section{Edición Extraordinaria. p.p. 1125 - 1136}

Memorias del IX Encuentro Nacional de Experiencias en Enseñanza de la Biología y la Educación Ambiental. IV Congreso Nacional de Investigación en Enseñanza de la Biología.

cada una de las instituciones de la comunidad mencionadas al inicio de este documento. Los derechos de petición fueron entregados a conservación Internacional para que fueran radicados.

Mientras que iban y venían los documentos mencionados, la situación se agravó en la primera semana de noviembre de 2016, periodo en el que las lluvias estuvieron presentes en los cerros orientales de la ciudad de Bogotá, han ocurrido dos deslizamientos desde la montaña de escombros sobre la quebrada Puente de Piedra, corriendo el cauce de la misma por lo menos 3 metros más en dirección a la escuela. Ante esta situación se hizo un llamado a diferentes instancias distritales como los Bomberos, IDIGER ${ }^{8}$, La CAR, Aguas de Bogotá Y Acueducto de Bogotá, éstas han venido a revisar la situación y los informes técnicos emitidos por la CAR el 30 de diciembre de 2016, solo describen la situación:

Se presenta un fenómeno de remoción en masa que involucra un depósito de aproximadamente $45.000 \mathrm{mts}^{3}$ de residuos sólidos heterogéneos, ubicados en un área de $3600 \mathrm{mts}^{2}$, cuya zona más elevada se encuentra sobre la ronda hidráulica y cauce de la quebrada Puente Piedra (...) dicho fenómeno fue causado por la elevada pendiente y la gran altura de $20-35 \mathrm{mts}$ alcanzada por residuos sólidos depositados en la zona la quebrada. (CAR, P.12)

Además de lo anterior remiten a otras entidades; sólo AGUAS DE BOGOTÁ se encargó de llevar a cabo un proceso de despápele (limpieza) sobre la quebrada y EL ACUEDUCTO ha mencionado que tratará de iniciar la gestión para construir un muro de contención al borde de la quebrada.

Después de estos acontecimientos, y en medio del pesimismo que nos embarga hasta enero del siguiente año 2017, comenzaron a llegar respuestas de los derechos de petición por parte de distintos entes, lastimosamente, se repitió el hecho de siempre, las entidades nuevamente remitieron los derechos de petición a otras entidades y en una de las reuniones realizadas con Conservación Internacional en la escuela, tristemente se observó que todo el trabajo realizado por esta entidad en años anteriores ha sido perdido, por ello las entidades participantes, se citaron en la EPE que el 17 de mayo, representantes de las distintas entidades estatales (CAR, Secretaria Distrital de Ambiente, alcaldías locales de Usaquén y chapinero, alcaldía de Bogotá, personería de Bogotá, IDIGER, policía nacional ambiental, Veeduría Distrital) que tiene competencia en este acontecimiento.

Esta reunión tuvo por objeto, que las entidades asumieran la responsabilidad ante el riesgo,

\footnotetext{
8 Instituto Distrital de Gestión de Riesgos y Cambio Climático: entidad encargada de Coordinar, orientar y consolidar el Sistema Distrital de Gestión de Riesgos y Cambio Climático -SDGR-CC y promover políticas, normas, planes, programas y proy ectos de gestión de riesgos y cambio climático para la transformación cultural, social y territorial del Distrito Capital, desde un enfoque de derechos y de sostenibilidad. Recuperado de: http://www.idiger.gov.co/mision-y-vision 24 de may o de 2017
} 


\section{Bio - grafía. Escritos sobre la Biología y su Enseñanza. ISSN 2027-1034}

\section{Edición Extraordinaria. p.p. 1125 - 1136}

Memorias del IX Encuentro Nacional de Experiencias en Enseñanza de la Biología y la

Educación Ambiental. IV Congreso Nacional de Investigación en Enseñanza de la

Biología.

y pactaran acuerdos de solución ante esta problemática. Al inicio se les presentó un panorama de los antecedentes jurídicos, las acciones comunitarias y procesos de restauración ecosistémicos llevados a cabo por la escuela, la comunidad junto con las organizaciones como Conservación Internacional; también se les mostró el estado actual de la quebrada, donde gran parte del trabajo de recuperación y restauración realizado en años anteriores se ha perdido, transformándose en una montaña de escombros que representa un riesgo para la comunidad EPE.

Como segundo momento se dieron varias intervenciones por parte de las entidades competentes, donde volvieron a pasar la responsabilidad a las otras; ante esto varios estudiantes se manifestaron haciendo un llamado a la reflexión recordándoles los derechos constitucionales y sus deberes respecto al cuidado y preservación del ambiente. Finalmente, acordaron reunirse nuevamente el 23 de mayo de 2017 para tomar decisiones, este acuerdo fue documentado por medios de comunicación como el periódico Las dos orillas y citytv, para que lo que se pactaron y que ahora se pretende acordar, no queden en palabras y mucho menos en el olvido.

La montaña de escombros es una realidad, no un imaginario que busca pasar por desapercibido en la medida que no se considera un problema para los cerros orientales, para las fuentes hídricas que la atraviesan, y para una escuela que se encuentra bajo el corazón de esta montaña.

\section{A MANERA DE CIERRE}

La realización de estos procesos nos demuestra que, trabajar desde las problemáticas cercanas a la escuela, nos permite el abordaje de las disciplinas, comprender conceptos biológicos, políticos, geográficos y sociales; y por consiguiente movilizar a los estudiantes y la escuela para promover la articulación con el barrio y diferentes instituciones con el fin de generar soluciones reales y aportar a la transformación del contexto.

A través de este proceso de los escombros en la EPE, podemos afirmar se ha logrado con los estudiantes un acercamiento a las problemáticas cercanas que conlleva transformar las relaciones con la naturaleza, con la comunidad y con nosotros mismos esto aún no termina. Si bien es cierto se ha posibilitado reconocer lo local como un tejido de relaciones con el otro que generan o permean la participación, donde se concretan acciones, pactos y hechos que requiere de los sujetos actitudes ciudadanas participativas y transformadoras en pro de las comunidades como lo plantea el maestro Rendón (2011):

Lo local se convierte en el lugar de la vida, donde se generan las relaciones cotidianas, el hacer, el sentir, el pensar, el desear, en fin, lo local es el espacio vital pero es también la oportunidad para la construcción social del territorio, de la reivindicación del desarrollo como derecho esencial (p. 3).

Es desde aquí que esta interacción con la comunidad de la UPZ 89 se ha generado la posibilidad de reconocer al Otro, un par con el que conversa, discute y establece responsabilidades, porque se reconoce que existen sabidurías propias y particulares que durante generaciones han permitido resolver problemas del día a día fundamentándose 


\section{Bio - grafía. Escritos sobre la Biología y su Enseñanza. ISSN 2027-1034}

\section{Edición Extraordinaria. p.p. 1125 - 1136}

Memorias del IX Encuentro Nacional de Experiencias en Enseñanza de la Biología y la

Educación Ambiental. IV Congreso Nacional de Investigación en Enseñanza de la Biología.

en un conocimiento popular, localizado, heredado y enriquecido cotidianamente porque está cargado de valores, proyectos de vida, metas alcanzadas y sueños por construir (Castellanos e Hinestroza citado por Alarcón 2016: 77).

Sin embargo, aún estamos a medio camino porque ahora seguimos cercados por una montaña de escombros ilegales que se ha convertido en un riesgo para la comunidad educativa. Esta situación ha sido denunciada a través de diversos medios de comunicación y a las organizaciones locales y nacionales como Personería, Contraloría, CAR; Secretaria de Ambiente; Alcaldía de Usaquén, IDIGER Y Empresa de Acueducto de Alcantarillado de Bogotá dado que acabó con el proceso de restauración del corredor ecológico de la quebrada Puente Piedra que se había adelantado con el apoyo de Conservación Internacional. Queremos ser optimistas, creemos que todavía hay mucho por hacer, existen voluntades que se han congregado para darle continuidad a este proceso que requiere de la participación de múltiples voces para rescatar nuestros cerros y quebradas en la ciudad de Bogotá... voces que emergen de tradiciones culturales y populares que destacan la necesidad e importancia de nuestros recursos así como de su cuidado y conservación, aspectos que desde la escuela se abordan en el marco del proyecto de Economía Azul, donde la idea de sustentabilidad se basa en encontrar un aprovechamiento, cuidado, uso y reconocimiento de aquellos recursos que nos rodean como una alternativa para el reconocimiento y valoración de lo vivo y la vida, y así mismo, de la enseñanza de la biología en diferentes contextos.

Por ello, la EPE es una escuela que posibilita vivenciar de diversos modos lo que es la sustentabilidad, y cómo desde este punto se pueden articular campos de saber y conocimiento que dan lugar a realizar un ejercicio constante de reflexión, análisis y problematización de aquellas situaciones ambientales que constantemente inciden en las dinámicas propias de este contexto escolar.

Es así, que la escuela está en el corazón de la montaña. De ella depende que el agua que corre por la quebrada Puente Piedra siga su curso; que su estabilidad y control, den seguridad a la comunidad educativa; que su crecimiento por cuestiones de contaminación e incidencia antrópica no generen amenazas en el territorio... ese es el corazón de la $E P E$, una montaña que busca liberarse del uso y abuso de escombros que a diario son depositados en ella.

\section{FUENTES BIBLIOGRÁFICAS}

Alarcón P. (2016). Otras Epistemologías: Conocimientos y Saberes locales desde el Pensamiento Complejo.

Cañal, P. (2004), la enseñanza de la biología ¿cuál es la situación actual y qué hacer para mejorarla? Revista Alambique didáctica de las ciencias experimentales, (41), 2741.

CAR. (30 de diciembre de 2016). Informe técnico No. DRB244. RESERVA FORESTAL BOSQUE ORIENTAL DE BOGOTÁ. Deslizamiento Quebrada Puente Piedra, km 4.5 Bogotá - vía la Calera. 
Bio - grafía. Escritos sobre la Biología y su Enseñanza. ISSN 2027-1034

Edición Extraordinaria. p.p. 1125 - 1136

Memorias del IX Encuentro Nacional de Experiencias en Enseñanza de la Biología y la

Educación Ambiental. IV Congreso Nacional de Investigación en Enseñanza de la Biología.

(Noviembre, 2016) I Foro Distrital abierto y amplio: Uso público de los cerros orientales de Bogotá, Universidad Santo Tomás, Bogotá, Colombia

García, M. (octubre, 2013).una escuela más allá de la escuela: proyecto de recuperación de la quebrada puente piedra. VII Encuentro Nacional de Experiencias en la Enseñanza de la Biología y la Educación Ambiental y II Congreso Nacional de Investigación en la Enseñanza de la Biología. Bogotá.

García-Mayorca, J.; Lujan, J.; Arbeláez, J.; Fernández, P.; Velazco, A.; \& García, M. (2014). Nuestro Entorno. Líneas de trabajo. Escuela Pedagógica Experimental. Bogotá - Colombia.

Guerrero, L (productor). (2016). Cimavision [ ]. Ciudad: Bogotá. Recuperado de: https://www.youtube.com/watch?v=bxeeidPuAcM

Molina A, Pedreros R. \& Segura D. (1997). Actividades de Investigación en la clase de ciencias. Sevilla-España. Editorial Diada.

Pedreros R., Velasco A., Leuro R. Arcos F., Hernández G. Molina A. \& Segura D, (1995). Vivencias de Conocimiento y Perspectivas de Cambio Cultural. Escuela Pedagógica Experimental. Colciencias. Bogotá - Colombia

Rendón, J. A. (2011) Centralización. Construcción Territorial y Desarrollo Local.

Ruiz, D; Granados, M \& Soto, R. (2016). Recuperación de la Quebrada Puente Piedra. Contaminación del entorno. Ponencia presentada en el III congreso intercolegiado de ciencias naturales.

Segura, D. (2007). Las Actividades Totalidad Abiertas, una propuesta para la comprensión de nuestra realidad en un mundo globalizado. Exploraciones fuera y dentro del aula. Ponencia presentada en IX Congreso Nacional de Ciencias. Agosto, Costa Rica.

Segura, D. (2008). Las urgencias de la innovación. 10ํㅡㄹ Congreso Nacional de Ciencias y Estudios Sociales. Costa Rica. Recuperado de: www.scribd.com/doc/8093805/Dino-Segura.

Velazco, A. (2001). Los cuestionamientos de los estudiantes como punto de partida en la realización de un proyecto educativo ambiental. Universidad pedagógica Nacional, Bogotá, Colombia.

García, M., Segura, D., \& Diaz, V. (2015). La educación ambiental en la escuela pedagógica experimental: hacia una versión pedagógica de la economía azul. Nodos y Nudos, 4(38), 25-36. 
Bio - grafía. Escritos sobre la Biología y su Enseñanza. ISSN 2027-1034

Edición Extraordinaria. p.p. 1125 - 1136

Memorias del IX Encuentro Nacional de Experiencias en Enseñanza de la Biología y la Educación Ambiental. IV Congreso Nacional de Investigación en Enseñanza de la Biología.

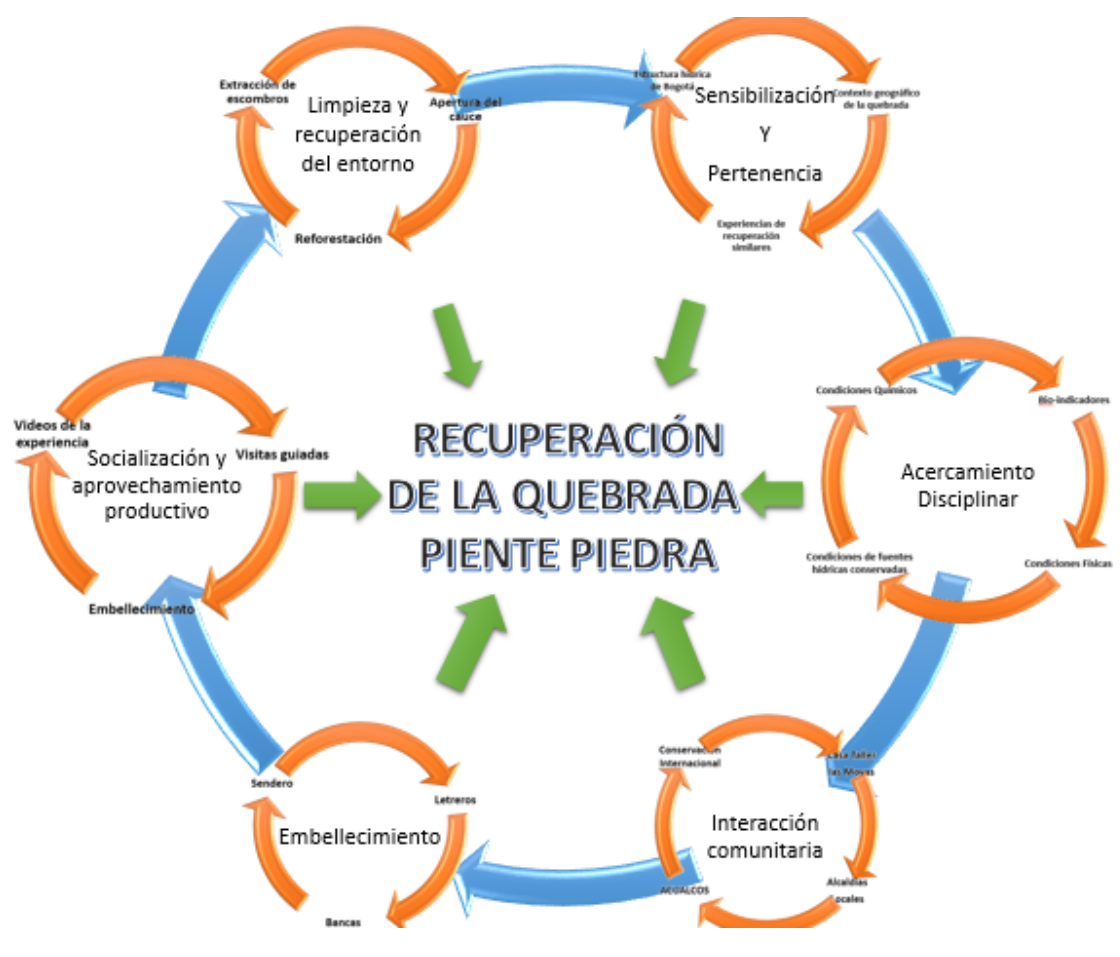

Figura 1. Esquema del desarrollo del proyecto de acuerdo a los ejes. 